\title{
Digital Caring: New Digital Divide and Response -- Observing Taiwan Distance Learning Companions Are the Basics of Social Stratification Perspective
}

\author{
Hong-Yen Lin ${ }^{1, ~ *}, \mathrm{Hu} \mathrm{Ke}{ }^{2}$ \\ ${ }^{1}$ Dept. of Information Engineering and Computer Science, Fu Jen Catholic University, New Taipei, Taiwan \\ ${ }^{2}$ School of Education, MinZu University of China, Beijing, China
}

Email address:

hylin@mail.fju.edu.tw (Hong-Yen Lin), hukeflame99@126.com (Hu Ke)

\section{To cite this article:}

Hong-Yen Lin, Hu Ke. Digital Caring: New Digital Divide and Response -- Observing Taiwan Digital Program Partners Are the Basics of Social Stratification Perspective. Humanities and Social Sciences. Vol. 3, No. 5, 2015, pp. 271-275. doi: 10.11648/j.hss.20150305.27

\begin{abstract}
There is a "digital divide" which is a gap in the information technology usage in different regions and between different groups and individuals. With the rapid development of information technology, digital divide gradually spread not only from the usage and access of information technology but also to the information knowledge and the new changes from the first level to the third level. However, it's not just limited to this, but after the emergence of "Internet+" having been integrated into our social life, the digital divide has affected people's learning habits and lifestyle, and even changed the individual hierarchical status in the society. Taiwan has promoted a series of initiatives to reduce the gap in the digital divide, and we have achieved several goals, especially with the distance learning companions together with the more humanism concern approach. This article tries to analyze the new change in digital divide from the information technological background, and from the perspective of social stratification theoretical analysis on digital divide and exploring how to care for the vulnerable. Besides, having recognized as "The project of distance learning companions" through caring for the weak and vulnerable in the society try to reduce the gap in digital divide more effectively.
\end{abstract}

Keywords: Digital Divide, Social Stratification, Distance Learning Companions, Humanism Concern

\section{数字关怀: 数字鸿沟的新变化及应对一一基于社会分层理论视角 看台湾数位学伴计划}

\author{
林宏彦 ${ }^{1, *}$, 胡科 \\ ${ }^{1}$ 资讯工程学系, 辅仁大学, 新北市, 台灣 \\ 教育学院, 中央民族大学, 北京市, 中国
}

邮箱

hylin@mail.fju.edu.tw（林宏彦）, hukeflame99@126.com（胡科）

\begin{abstract}
摘要：信息技术在不同地区和不同群体及个人之间的表现，出现了 “数字鸿沟(digital divide)”。随着信息化的发展， 数字鸿沟逐步从信息技术接入和使用的差距发展到信息知识的差距,即第一道沟到第三道沟的新变化。但不仅止于此, 当 “互联网+” 这一概念思维被融入到人们的社会生活之后, 数字鸿沟已经影响到人们学习习惯和生活方式, 进而改变 个人在社会中的层级结构。台湾推动了系列缩减数字落差的举措, 取得了一定了成效, 尤其是数位学伴计划更具人文 关怀色彩。本文试分析信息化背景下数字鸿沟的新变化, 并从社会分层理论视角分析数字鸿沟, 并通过 “数位学伴计 划” 在缩减数字落差的实际效用, 探讨如何关怀数字弱势、缩减数字鸿沟的应对策略。
\end{abstract}

关键词: 数字鸿沟, 社会分层, 数位学伴, 人文关怀 


\section{1. 引言}

2015年5月，联合国教科文组织与中国政府共同举办 的首届国际教育信息化大会通过成果文件《青岛宣言》， 站在信息化的角度重新审视 “教育公平” , 挖掘世界 “教 育公平” 的新内涵 [1]。揭示了信息化时代造成教育不公 平的 “新问题” - “技术和在线内容的不易获取正在增 大长期存在的学习鸿沟”。近年来, 信息技术在教育领域 内推陈出新, 大规模开放在线课程 (Massive 0pen 0nline Course，简称MOOC或慕课）在全球迅速升温，信息技术与 教育进入深度 “融合” 阶段, 教育进入信息化时代。利用 信息技术促进教育公平与发展成为各地区的社会议题。信 息化可以是教育公平的助推器, 但 “数字鸿沟” 却有可能 使这些努力成为徒劳, 甚至加剧 “教育不公平”。从这个 角度说来，《青岛宣言》也意在缩小 “数字鸿沟”，以 “信 息公平”促进教育公平。

然而, 信息技术发展越快, 数字鸿沟也会伴随着扩 大。从技术的接入到使用差异的不平衡, 再到信息资源 和知识方面的差距, 已经造成了不同地区之间、不同群 体之间及个体之间的差异, 进而影响个人的学习方式和 社会资源的获得, 从而对社会阶层流动产生影响。但缩 减数字鸿沟并非一蹴而就, 甚至越来越难消除。因此只 有结合不同层级信息需求, 逐步缩小差距, 从而达到数 字鸿沟向数字机遇的转变, 力求做到机会公平, 而非均 衡发展。

基于 “缩减城乡数位落差” 的政策, 台湾行政院 2002年发布了《挑战2008: 国际发展重点计划书（2002 -2008)》。其中第六项为《数位台湾 (e-Taiwan) 计划 书》, 2004-2007年又提出 “缩短城乡数位落差”, 在 2008 －2011年间实施 “创造偏乡数位机会”，2012-2015年 “提升数位人文关怀” [2]。在《新世纪第三期国家建设 计划（2009-2012）》之教科文建设之教育篇的规划策 略中, 将 “强化弱势扶助, 缩短城乡差距, 均衡资源分 配” , 列为 “公义关怀” 执行要项。规划 “招募大专校 院志工在线课后辅导, 服务偏乡弱势学童” [3]。远距教 学早已有多年的历史, 但在缩减数字落差方面收效甚微, 而数位学伴计划 “用生命陪伴生命; 以生活教导生活”

(吕慈涵, 2014) [4]作为其核心理念, 赋予了远距教学 新的内涵和发展。

\section{2. 社会分层理论下的数字鸿沟}

1989年英国《时代教育专刊》发表了一篇题为 “数字 鸿沟” 的文章, 这是首次使用数字鸿沟这一概念。但直到 1995年美国政府发布 “Falling through the Net” 的研 究报告使这一术语开始流行 (Servon, 2002; NTIA，1995), 研究也从其内涵扩展到信息公平和社会不平等等领域。数 字鸿沟的内涵变化同信息技术发展变化相一致, 是一个动 态扩展的过程。

\section{1. 数字鸿沟的新变化: 信息鸿沟}

数字鸿沟的发展经历了三代: 在最初阶段, 数字鸿沟 主要关注拥有者和缺乏者在接入信息通讯技术方面的鸿 沟, 接入主要是是否具备相应的硬设备、信息环境和接入 管道; “第二代” 数字鸿沟, 发展到包括 ICT 素养和培训、 ICT 利用水平方面的鸿沟 (Joel Cooper, 2003) [5]。如 伊利诺大学情报学教授凯特 - 威廉姆斯 (Kate Williams) 把数字鸿沟定位在 “社会鸿沟”, 体现在接入和利用计算 机和计算机方面拥有者和缺乏者的社会差距 [6]; “第三 代” 数字鸿沟的研究, 即关于数字时代信息资源和知识的 鸿沟。侧重于 “信息与知识获取和利用能力的差距” (赖 茂生，2000）[7]，韦路等人认为 “第三道数字鸿沟” 是 “知识获取鸿沟” [8], 这一时期的数字鸿沟关注信息资 源的拥有和缺乏。

上述 “三代” 数字鸿沟从信息的接入、使用逐步到信 息知识的扩展, 丰富了数字鸿沟的内涵。但信息技术仍在 不断的发展变化, 并与传统行业结合衍生发展, “互联网 +”思维将传统的课堂与信息技术结合起来, MOOC的形成 就已经证明了数字鸿沟已经进一步扩展, 进入了 “信息鸿 沟”。在信息化社会里, 当信息接入、使用和信息资源都 能够满足时, 数字鸿沟就体现在学习能力的差距上, 即如 何运用有效的学习方式去融合理解数字信息。信息素养关 注信息的意识、获取、评估及创造等方面, 缩小信息鸿沟 就是要基于信息素养构建数字化时代的元素养 [9]。

\section{2. 数字鸿沟的分层结构}

社会分层是指社会成员在社会生活中由于获取社会 资源的能力和机会不同, 而呈现出高低有序的等级或层次 的现象和过程（刘祖云, 2002)。随着 ICT 的快速发展, 人们逐渐发现互联网技术中的梅特卡夫定律 (Metcalf' s Law ${ }^{\circledR}$ 并不完全适用于现实情况。用户的质量而非数量的 影响力逐渐扩大, 数字化进程表现出明显的不平衡状态。 例如, 世界收入最高和最低的五分之一人口分别拥有互联 网用户的 93\%和 $0.12 \%$ （刘芸，2007）。对于数字鸿沟有 根据不同的情况有不同的需求, 基于对社会分层理论的理 解, 将数字鸿沟的层级分为四个方面:

（1）数字环境需求层一接入鸿沟: 主要是偏远贫困 山区的居民、身心障碍者、弱势儿童等, 处于经济弱势, 信息接入条件简陃, 信息环境缺乏, 客观环境条件及个人 生活处境不易使用数字资源, 是信息化社会的边缘地区和 群体。

（2）数字运用需求层一使用鸿沟: 农村中年群体、 基层务工人员、偏远山区中小学生等, 基本了信息技术的 使用条件, 也有相应信息技术设备, 如手机、计算机等, 基础数字环境完善。但是因缺乏信息素养和信息技术使用 知识, 无法有效的利用数字资源, 提升生活质量。

(1)互联网发展的早期定律之一: 网络价值等于网络用户数的平方, 它强 调了网络经济与传统经济边际效益规律的不同, 从而突出网络的扩散性 与外部性。 
（3）数字整合需求层一知识鸿沟: 城市地区居民、 中青年工薪阶层等, 数字使用环境完善, 并能够便捷的获 取数字资源, 具备一定的信息素养。需进一步将信息整合 分析、转化成知识, 以实现信息共享和信息流畅使用。

（4）数字创造需求层一信息鸿沟: 高级知识分析、 社会精英阶层等, 具备专业信息素养, 有丰富的信息资源, 并能够创新的转化为数字成果, 能够有效、负责任的利用 信息为提升自身生活质量服务, 并能够对其他数字弱势阶 层进行关怀。

\section{3. 台湾数位学伴计划的理念及其运作}

2006年台湾地区开始推动《偏乡地区中小学网络课业 辅导服务计划》, 运用网络教学方式, 由大学生辅导偏乡 地区的国中、小学学生, 提升偏乡学生学习成效, 促成学 习机会均等。2007年进一步将网络课业辅导服务扩展至北 部及南部偏远地区之学校。2008-2009年更扩大结合东部 持续办理, 并加入计算机受赠户学生及数位机会中心 (Digital Opportunity Center, 简称DOC) [10]邻近学生, 共同协助提升偏远地区中小学学生的教育、文化及信息素 养, 以缩减城乡学习差距, 提升当地教育质量。2010年命 名为 “数位学伴线上课业辅导服务计划” , 实施迄 2013 年1月底, 已辅导学童人次 7005 , 培养大学课辅老师 10875 人次、累积上课时数 340058 小时 $[11]$ 。往后两年依核定在 案的计划, 每学期学童以 1200 人次、大学课辅老师以 2000 人次的数量稳定运作。

\section{1. 数位学伴的运作模式}

计划以“用生命陪伴生命, 以生活教导生活”为理念, 将信息技术与人文关怀结合在一起。计划运作机制, 教学 端与学习端, 都需要建置计算机、网络环境。召募大批大 学生担任课业辅导老师, 以集体聚集教学及学习为执行核 心运作模式。教学端的大学生及学习端的中小学生, 皆汇 集于各自校内计算机教室, 采一对一上课方式, 可避免双 方在家联机, 缺乏后端管理, 两人整晚都在闲聊的虚耗, 整个计划运作效能, 因而大幅提升。教学平台采视频会议 系统, 必须能够承载瞬间庞大流量为基本条件。此外, 还 需要对负责教学辅导的大学生, 进行严格的甄选、培训; 建立管理系统、伙伴关系经营等配套措施 (林宏彦、杨志 田, 2012) [12]。

从数位环境需求来说, 数位学伴计划需要计算机硬件、 软件、视讯设备 (耳麦、Webcam) 、系讯系统、网络等条 件。而从数位运用与创造来说, 数位学伴计划中最重要的 是人员的素养部分, 数位学伴计划之所以不同于以往的网 络课辅, 就在于以一对一的关怀和陪伴。现在的网络课辅 已经变化多样, 有以课程视频为主、也有以MOOC形式的, 但这些网络课辅需要有较强的自主学习能力。对于绝大数 中小学生尤其是弱势学业的学生来说, 缺乏自主学习能力 是学业低成就的重要因素。而要想达到辅导效果, 网络课 辅就需要即时的互动和回馈。数位学伴计划就是一对一的 即时教学模式。在这个计划中需要对课辅教师甄选, 并进 行教育训练, 同时借助教学平台和教学日志管理系统进行
监督、改善和提高。同时建立大学伴与大学伴之间、大小 学伴之间、大学合作伙伴学校之间、大学与社区或中小学 之间的合作关系, 全方位的推动数位学伴计划, 展现社会 对弱势孩童的关心。

\section{2. 数位学伴计划的多重效应}

计划的研究学者通过对大学生发放问卷分析, 研究发 现大学生参与数位学伴计划在利他、服务方面有正向成长, 对大学生在人格、品德方面有显著成效（Lih-Juan ChanLin, Hong-Yen Lin, Tze-Han Lu, 2012) [13]。林羿 瑄及刘旨峰教授等经由研究发现远距教学效果是有效的

（林羿瑄等, 2014）[14]。可见, 就实际成效来说, 数位 学伴的推行具有多重效应。

首先是大小学伴相互成长, 大学生透过计划培育, 提 升他们之认知、行动、反思能力, 培养具社会关怀与服务 胸襟之社会青年（如表1）。在参与这个计划中去了解偏 乡地区弱势孩童的处境, 关注台湾社会的不平衡发展, 在 教学的过程中能够不断的增进学习和教学经验, 这对于大 学生涯和大学生人格特质的培养具有一定的积极作用; 中 小学学童在线课业辅导与陪伴, 透过计划的执行, 协助他 们建立在学科上具备自主学习能力, 及自信与健全之人格。 大学伴是小学伴的典范, 小学伴通过向大学伴学习, 也能 够获得更多的外界文化刺激, 了解外界的社会动态, 还能 够对学业有所帮助, 这对于引导好小学伴朝健康积极的方 向发展大有裨益。其次是大学参与, 大学具有丰富的资源, 也应该承担社会服务的责任。企业属于社会主体的一部分, 也要承担企业社会责任。通过参加该计划, 企业与大学合 作, 将资源与关怀传给偏乡弱势地区, 能够缩减数位落差, 推动社会发展。最后是利于整个社会资源的整合, 有助于 推动数位公益。数位公益是以人文关怀为核心精神, 运用 信息技术，搭建公益平台，为教育资源不足之地区、家庭 学童, 开创新的学习模式, 弥补环境或先天条件之不足。 使之, 有公平学习机会。（林宏彦, 杨志田, 2013)

表 1 大学生在数位学伴计划中的能力培养 [15]。

\begin{tabular}{ll}
\hline 策 略 & 方 法 \\
\hline & 1) 团队由全校各系同学组成, 各种领 \\
& 域都有, 计划进行过程有许多相互交流 \\
六力: 全球移动力、就业力、机会 \\
创新力、跨域力、资讯力、公 & 2) 资讯工程系负责, 动员系上师资、 \\
民力 & 设备、环境, 提供最新资讯知识、技能 \\
& 3) 以资讯为基础, 人文关怀为核心, \\
& 学习关怀弱势 \\
5C: 沟通能力 & 1) 每位学童配置两位老师, 语文 (国 \\
(Communication)、团队合作 & 英)、自然 (含数学), 跨领域沟通 \\
能力 (Collaboration)、反思 & 2) 小组讨论教学与辅导方法, 训练团 \\
能力 (Critical thinking)、保合作与沟通能力 \\
解决复杂问题能力 (Complex \\
problem solving)、创造力 \\
(Creativity)
\end{tabular}

\section{3. 社会分层与数位学伴计划}

从社会分层理论来看数位落差分层情况, 数位学伴整 体运作, 各环节恰如本文所撰述的四个典型需求层: 
(1) 数字环境需求层: 偏远地区中小学学童的学习需 求。偏远地区教育环境与城市间有鸿沟, 以致有补救教学 的需求; 外界藉由数字科技, 克服遥远距离一空间因素、 不能即时或随时施予援助一时间因素的藩篱, 将教育资源 藉由信息技术, 源源不断输入;

(2) 数字运用需求层: 大学伴运用数字科技辅导偏乡 学童。设计教材, 在线教学、陪伴, 填补偏远地区学童的 学习需求;

(3) 数字整合需求层: 大学与中小学师长整合双方资 源。争取校方支持, 联合培训大学生辅导与在线教学技能, 善用计划所需之人力、物力, 发挥高度效能;

(4) 数字创造需求层: 教育部创造数位学伴计划。了 解偏远地区中小学学童学习上的困境和大学拥有之能量 资源, 邀请各方相关单位参与, 共同努力为偏远弱势族群 服务。

将近十年的计划运作, 数位学伴计划中数位环境需求 层已经得到基本的满足, 在中华电信等企业的支持之下, 偏乡地区的信息技术设备得到了保障和补充。在数位运用 需求层中，设置的数位机会中心（DOC）能够帮助当地居 民学习, 对当地的文化进行数位典藏。可以说, 前面两个 层级的满足是台湾推行缩减数位落差举措的重要成果。数 位学伴计划的推出进一步实现了数位整合需求层的满足, 通过大学生与小学生的一对一辅导, 一方面大学生在备课、 授课的过程中不断的运用信息技术设备达到满足, 并对课 本知识内容加以创造, 传递给小学伴; 另一方面小学伴通 过大学伴的教导, 也逐渐从基本信息技术的使用转移到运 用工具促进自己学习的目标上来, 提高了学生的信息素养。 而当下数位学伴追求人文关怀、数位公义, 就是对第四层 数位创造需求层的满足。在这一层级上, 加入了 “以人为 本” 的教育理念, 从人性的角度出发, 不论社会处于那一 阶层、不论贫穷与富贵、男女老弱, 都有能够有使用数位 技术学习的机会, 并能通过信息技术作为终身学习的延伸 工具, 从而达到整个地区以及全球的数位包容。

\section{4. 数字关怀: 缩减数字鸿沟的分层策略}

缩减数位落差, 促进教育公平公正已经成为全球议题。 台湾数位学伴计划将信息技术与人文关怀有机结合, 对网 络课辅进行了实质性的革新, 成为了数位公义的典范。缩 小数字鸿沟, 不仅仅是数字环境的建立, 更多的应该是人 文关怀的投入。但这一过程并非一蹴而就, 而是要根据偏 乡弱势的实际需要, 分层推进, 逐层缩小。从数字鸿沟的 层级来看, 可以分为两个阶段四个层级来满足。

\section{1. 关怀初阶一数字环境与运用的满足}

数字鸿沟的出现，是信息技术发展带来的结果。数字 环境的需求是数字关怀的基础, 只有具备了一定的数字环 境, 才能够去进行培训和运用。

对于数字环境需求层来说, 首要条件是创建数字化环 境, 包括网络、计算机、手机等设备的接入, 政府要加大 财政投入, 制定缩减数字鸿沟的政策, 落实具体行动。如 使得偏远山区居民手机信号无死角、村村能够接通宽带或
者无线信号，在各数字弱势地区设立数字服务中心等等。 要升级更新已有的数字资源, 建立活化的数字环境, 针对 特殊弱势族群, 要结合社会资源, 提供个人化的数字环境。

对于运用需求层来说, 关键是要教会人们如何利用已 有的数字环境, 现在中国大陆实施了 “三通两平台” 的项 目, 即推动宽带网络校校通、优质资源班班通、网络学习 空间人人通，搭建教育资源公共服务平台和教育管理公共 服务平台。但具备了这些数字化条件, 如果不能够有效的 利用也会造成资源的浪费。在一些偏远山区，依靠社会力 量建立了多媒体教室或信息服务中心等, 但因为不会使用 而未发挥作用。因此, 要加强数字设备使用技能的培训, 提供线上线下的学习服务。

\section{2. 关怀进阶一数字整合与创造的满足}

数字环境建置是关怀初阶的基础, 数字流畅的运用是 数字公民的必备能力。在 “大数据” 时代, 简单的运用已 经不能够满足对数据的分析需求。有效的数据提取和分析 需要对资源进行整合, 进而才能够去发挥和创造出新的成 果, 但这种成果是 “以人为本” 的, 缺乏人文关怀的数字 创造只会是冰冷的产品。

对数字整合需求层来说, 要培养信息素养, 包括信息 需求、获取、评价、组织与交流等能力, 加强小区、学校 信息素养教育人才之培育, 以稳定信息教育师资, 提供公 平信息使用机会, 持续关注偏远地区的信息素养, 提升网 络使用普及率，主动向偏远地区、弱势团体、原住民与妇 孺、年老退休人员等提供所需要的数字信息, 提供使用网 络及教育训练的机会。并整合多元化数字资源, 确保全体 民众都能获得公平的信息与学习机会, 进而提升全民的信 息素养。

对数字创造需求层来说, 要提升数字创新、创造使用 的能力, 转向元素养的培养, 提升数字生活内涵。在相关 政策基础上, 由量的变化扩展到质的深化, 达成促进族群 多元应用, 迈入更为广泛和深化的数字关怀。继续提升中 国偏远地区网络建设和弱势族群信息环境, 开设提升弱势 族群元素养与教育课程, 推动数字资源和生活质量的发展, 积极发展数字公正, 均衡不当使用数字资源的现象, 落实 高等教育在数字研究计划和资源运用上的社会责任。

\section{5. 小结}

借助信息技术促进教育公平已经成为一条既定政策 被大力推行, 并以此为基础进而探索一些机制和操作层面 的问题。但实际效果证明, 数字鸿沟并没有缩减, 反而继 续加大的趋势。即便是教育技术研究者也承认, “在教育 技术的发展历史过程中, 教育技术研究者的承诺远大于实 际” [16]。之所以出现这样的现象, 一方面是由于信息客 观环境的发展所致, 另一方面也是由于缩减数字鸿沟政策 的不具体化。没有结合各层级数字鸿沟制定因地制宜的政 策, “一刀切” 的方法只会导致数字鸿沟的扩大。因此要 承认数字鸿沟的客观存在, 再结合不同层级逐步缩小, 才 能事半功倍。 
“数位学伴计划” 以学习端需求为导向之在线即时互 动机制: 充分与学习端师长、家长沟通、经验分享, 共同 规划在线陪伴与学习教学计划; 以计划资源与偏乡师长合 作, 激励偏乡教育典范, 创造社会价值; 整合 “教学、辅 导、品德”三合一在线陪伴与学习内涵, 落实陪伴与学习 之服务内涵; 实践大学社会参与, 大学拥有高等知识力量、 丰沛人力、新颖信息科技设备等, 藉由计划的运作, 服务 偏远地区学童, 补救过去未曾学会的学科知识、增加学习 能量、改善不良生活态度等。拉拔成功一名孩童, 即为社 会累积一份正向力量。大学生为了做典范给学童学习, 也 会主动调整个人不良习惯, 人生态度因而改变 [17]。数位 学伴计划正逐步从数位关怀初阶深入到数位关怀进阶, 这 种模式为缩小数字鸿沟提供了新的思路。

需要重申的是, 数字鸿沟将长期存在, 并难以消除, 数字关怀不是要达到教育公平, 而是要促进教育公正, 机 会均等。同时, 数字鸿沟可以相对缩小, 但行之有效的方 法不是一律的均衡数字资源, 而是有步骤的、分层级的进 行发展和关怀。

\section{致谢}

数位学伴计划, 由台湾教育部主办, 輔仁大學校方全 力支持; 计划成员有 20 多所大学, 每学期号召 2000 多名大 学生、近百所中小学或数位机会中心 (DOC) 每学期约 1200 名学童参与; 大家同心协力, 使得本计划逐年得以顺利推 动。谨此向所有参与单位及伙伴们, 敬致谢意!

\section{参考文献}

［1］来源：中国教育报。信息时代教育公平的新内涵一一国际 教育信息化大会《青岛宣言》解读 $[\mathrm{EB} / \mathrm{OL}]$. http://www. moe.gov.cn/jyb_xwfb/moe_2082/z1_2015n/2 015_z114/201506/t20150616_190525. htm1

[2] 臺灣教育部網站, 偏鄉數位關懷推動計畫, http://itaiwan. moe. gov. tw/Plan01. aspx

[3] 臺 灣 教 育 部 數 位 學 伴入口網, https://etutor.moe.gov. tw/edu_index/introduction_1 ist. php

[4] 輔仁大學, 臺灣偏鄉教育關懷中心網站, http://rural-caring. fju. edu. tw/web/
[5] Cooper J, Weaver K D . Gender and computers: Understanding the digital divide [M]. Mahwah: Lawrence Erlbaum Associates, Inc., publishers, 2003.

[6] Williams K. What is the digital divide? [OL]. A working paper for the d3 workshop, Ann Arbor, MI, August, 2001. http://www-personal. umich. edu / katewi11/kwd3workshop. pdf. 12/12/2007.

[7] 赖茂生。信息化与数字鸿沟 $[J]$. 现代信息技术, 2000 (12)。

[8] 韦路, 张明新。第三道数字鸿沟：互联网上的知识沟 $[\mathrm{J}]$. 新 闻与传播研究, 2006, 13( 4)。

[9] Mackey T P, Jacobson T E. Reframing Information Literacy as a Meta literacy. College \& Research Libraries, 2011(1): 62-78.

[10] 臺灣教育部偏鄉數位關懷推動計畫網站, http://itaiwan. moe. gov. tw/

［11］林宏彥，楊志田（2013）。科技應用與人文關懷合作提升 弱勢學童學習機會一臺灣數位學伴計畫。GCCCE2013全球華 人計算器教育應用大會, 工作坊: 數位公益。北京: 北京 大學。

[12］林宏彥、楊志田 (2012)。網路應用在偏鄉教育一遠距課輔 無遠弗屆一以數位學伴線上課業輔導服務計畫為例。輔仁學 志。第45期。第25-44頁。

[13] Lih-Juan ChanLin, Hong-Yen Lin, Tze-Han Lu. College students' service learning experiences from e-tutoring children in remote areas 4th WORLD CONFERENCE ON EDUCATIONAL SCIENCES (WCES-2012) 02-05 February 2012 Barcelona, Spain.

[14] 林羿瑄、劉旨峰、張琬羚、林俊閞。以帶班老師的觀點敘 說一對一遠距公益家教培養大學生數位素養與社會關懷懷。 第十八屆全球華人計算機教育應用大會 ( GCCCE 2014）, 2014，5月。上海華東師範大學。

[15] 林宏彥, 2014教育部數位學伴線上課業輔導服務計畫一輔 仁大學年度期末報告書, 2015, 1月。

[16] Merrill, M. D. \& Elen, J. (2014). A look forward. In J. M. Spector, M. D, Merrill, M. D. \& Elen, \& M. J Bishop (Eds), Handbook of research on education communication and technology ( $4^{\text {th }}$ Edition $)$ (pp. 873-874). New York, NY: Springer Science + Business Media.

[17] 林麗娟, 林宏彥, 呂慈涵。線上學伴服務大學生社會參與指 標之建構, 教育傳播與科技研究, 2014年8月, 108 期, 第 17-29頁。 